ESAIM: PROCEEDINGS AND SURVEYS, September 2014, Vol. 45, p. 88-97

J.-S. Dhersin, Editor

\title{
FORWARD IMPLIED VOLATILITY EXPANSION IN TIME-DEPENDENT LOCAL VOLATILITY MODELS ${ }^{*, * *, * * *}$
}

\author{
ROMAIN BOMPIS ${ }^{1}$ AND JULIEN HOK ${ }^{2}$
}

\begin{abstract}
We introduce an analytical approximation to efficiently price forward start options on equity in time-dependent local volatility models as the forward start date, the maturity or the volatility coefficient are small. We use a conditional expectation argument to represent the price as an expectation of a Black-Scholes formula computed with a stochastic implied volatility depending on the value of the equity at the forward date. Then we perform a volatility expansion to derive an analytical approximation of the forward implied volatility with a precise error estimate. We also illustrate the accuracy of the formula with some numerical experiments. Some results and tools of this work were presented at the conference SMAI 2013 in the mini-symposium "Méthodes asymptotiques en finance".

Résumé. Nous introduisons une approximation analytique afin d'évaluer efficacement les options à départ différé dites forward start dans les modèles à volatilité locale qui dépend du temps quand la date forward, la maturité ou le coefficient de volatilité sont petits. Nous utilisons un argument d'espérance conditionnelle pour représenter le prix comme l'espérance d'une formule de Black-Scholes calculée avec une volatilité implicite stochastique qui dépend de la valeur de l'action à la date forward. Ensuite, nous effectuons un développement en volatilité pour obtenir une approximation analytique de la volatilité implicite forward avec une estimation précise de l'erreur. Nous illustrons également la précision de notre formule avec quelques expériences numériques. Certains résultats et outils de ce travail ont été présentés au congrès SMAI 2013 dans le mini-symposium "Méthodes asymptotiques en finance".
\end{abstract}

\section{INTRODUCTION}

The values of certain path-dependent options, as the family of cliquet options, are sensitive to the anticipated level of volatility at some future date. The evaluation and the hedging of such products are far from trivial and the market has not yet settled for an agreed reference model. In this article, we focus on the pricing of forward start options, which constitutes the building block for more complex cliquet structures as the Napoleons, the multiplicative cliquets or the reverse cliquet (see [7]). A forward start option begins at some specified future date $t_{i}>0$, the forward date, and with an expiration further in the future $t_{i}+T$ with $T>0$, the premium being paid in advance at the initial date $t_{0}=0$. Denoting by $S_{t}$ the price at time $t$ of the underlying asset, we concentrate in this work on the payoff: $\left(\frac{S_{t_{i}+T}}{S_{t_{i}}}-K\right)_{+}$for a given strike $K>0$.

$\triangleright$ Literature review. Regarding the pricing of forward start options, it seems that many authors have mainly

* We thank Emmanuel Gobet for his constant support and encouragement.

** The first author research is partly supported by the Chair Financial Risks of the Risk Foundation.

*** We thank the referee for its remarks to improve the quality of this paper.

${ }^{1}$ CMAP, Ecole Polytechnique, Route de Saclay, 91128 Palaiseau cedex, France. Email: romain.bompis@polytechnique.edu

2 Markit, Ropemaker Place 25 Ropemaker Street London EC2YLY, UK. Email: julien.hok@markit.com

(c) EDP Sciences, SMAI 2014 
considered the case of models with stochastic volatility like the Heston model (see $[10,13,14]$ ) and the SABR model (see [9]), or the context of interest rates with the Hull-White model (see [6]). An alternative modeling is the use of Levy processes proposed for instance in [4]. Recently, Jacquier and Roome [12] provided an expansion formula of the forward implied volatility using calculations based on the forward characteristic function and large deviations techniques. Such an enthusiasm for the stochastic volatility models or more generally for two or more factors models in the literature can be explained by the potential availability of closed formulas using the (semi) explicit computation of the forward characteristic function owing to the tower property for conditional expectations. In the class of models mentioned above, we start with a price process or a joint process pricevolatility and deduce more and less the dynamic of the future volatility. To get a better control on the dynamic of the implied volatility, Bergomi modelises jointly the dynamics for the forward variance swap and the spot consistently and discuss about the calibration and pricing in $[2,3]$. To the best of our knowledge the case of local volatility models is not handled in the literature and the purpose of this work is to provide an accurate and tractable analytical approximation of the forward implied volatility in time-dependent local volatility models.

$\triangleright$ Formulation of the problem and our contribution. In this work, we consider financial products in a world with no interest rate written w.r.t. a single asset which price at time $t$ is denoted by $S_{t}$ paying no dividend. We consider a linear Brownian motion defined on a filtered probability space $\left(\Omega, \mathcal{F},\left(\mathcal{F}_{t}\right)_{t \geq 0}, \mathbb{P}\right)$ where $\left(\mathcal{F}_{t}\right)_{t \geq 0}$ is the completion of the natural filtration of $W$. We suppose that $S$ follows the local volatility model under the measure $\mathbb{P}$, i.e. it is the unique strong solution of the next SDE:

$$
\mathrm{d} S_{t}=S_{t} \sigma\left(t, S_{t}\right) \mathrm{d} W_{t}, S_{0}>0 .
$$

We assume that $S$ is a strictly positive martingale and we define the $\log$-asset $X=\log (S)$ which satisfies:

$$
\mathrm{d} X_{t}=a\left(t, X_{t}\right) \mathrm{d} W_{t}-\frac{1}{2} a^{2}\left(t, X_{t}\right) \mathrm{d} t, \quad x_{0}=\log \left(S_{0}\right), \quad a(t, x)=\sigma\left(t, e^{x}\right) .
$$

We are interested by the price at time 0 of a forward start call option written as:

$$
\operatorname{Call}^{\mathrm{FS}}\left(S_{0}, t_{i}, T, K\right)=\mathbb{E}\left[\left(\frac{S_{t_{i}+T}}{S_{t_{i}}}-K\right)_{+}\right]=\mathbb{E}\left[\left(e^{X_{t_{i}+T}-X_{t_{i}}}-e^{k}\right)_{+}\right],
$$

where $t_{i}>0$ is the forward date, $T>0$ the forward maturity, $K=e^{k}>0$ the strike and $\mathbb{E}$ stands for the expectation operator. Note that a change of probability measure argument allows to extend the results of the paper to products with payoff $\left(S_{t_{i}+T}-K S_{t_{i}}\right)_{+}$. If $S$ follows a log-normal model with deterministic volatility $\left(\sigma_{t}\right)_{t \in[0, T]}$, the price is explicitly given by $\mathrm{Call}^{\mathrm{BS}}\left(0, \int_{t_{i}}^{t_{i}+T} \sigma_{t}^{2} \mathrm{~d} t, k\right)$ where $\mathrm{Call}^{\mathrm{BS}}(x, y, z)$ denotes the Black-Scholes Call price function depending on $\log$-spot $x$, total variance $y$ and log-strike $z$ and defined by:

$$
\begin{aligned}
\text { Call }^{\mathrm{BS}}(x, y, z) & =e^{x} \mathcal{N}\left(d_{1}(x, y, z)\right)-e^{z} \mathcal{N}\left(d_{2}(x, y, z)\right), \\
\mathcal{N}(x) & =\int_{-\infty}^{x} \frac{e^{-u^{2} / 2}}{\sqrt{2 \pi}} \mathrm{d} u, \quad d_{1}(x, y, z)=\frac{x-z}{\sqrt{y}}+\frac{1}{2} \sqrt{y}, \quad d_{2}(x, y, z)=d_{1}(x, y, z)-\sqrt{y} .
\end{aligned}
$$

For the general case, instead of resorting to time-costing numerical methods like PDE techniques or Monte Carlo simulations, we aim at providing an accurate analytical approximation involving the same computational time than the application of the Black-Scholes formula. To achieve this, we start from the vanilla implied volatility approximation provided in [5, Theorem 22] and then we use a conditioning expectation argument to express the price (1) of the forward start option as an expectation of the Black-Scholes price function with a stochastic volatility argument involving the local volatility function frozen at $X_{t_{i}}$, plus an error. Then we perform a volatility expansion to consider the local volatility function frozen at the log-spot $x_{0}$.

The article is organised as follows. First we give in Section 1 some notations and definitions repeatedly used throughout the paper. Then we provide in Section 2 (see Theorem 2.1) a third order forward implied volatility expansion. Section 3 is devoted to numerical experiments illustrating the accuracy of our approximation. 


\section{Notations, DEFinitions And ASSUMPtions}

$\triangleright$ Assumptions on $a .\left(\mathcal{H}_{a}\right): a$ is a bounded measurable function of $(t, x) \in\left[0, T+t_{i}\right] \times \mathbb{R}$, and five times continuously differentiable in $x$ with bounded derivatives. Set

$$
\mathcal{M}_{1}(a)=\max _{1 \leq i \leq 5} \sup _{(t, x) \in\left[0, T+t_{i}\right] \times \mathbb{R}}\left|\partial_{x^{i}}^{i} a(t, x)\right| \text { and } \mathcal{M}_{0}(a)=\max _{0 \leq i \leq 5} \sup _{(t, x) \in\left[0, T+t_{i}\right] \times \mathbb{R}}\left|\partial_{x^{i}}^{i} a(t, x)\right| .
$$

In addition, there exists $\left.\left.\mathcal{C}_{a} \in\right] 0,1\right]$ such that $a(t, x) \geq \mathcal{C}_{a} \mathcal{M}_{0}(a)>0$ for any $(t, x) \in\left[0, T+t_{i}\right] \times \mathbb{R}$ (ellipticity condition).

$\triangleright$ Temporal shift of the local volatility function a. We introduce the time-shifted local volatility function $\alpha$ defined by $\alpha(t, x)=a\left(t+t_{i}, x\right)$ for any $(t, x) \in[0, T] \times \mathbb{R}$.

$\triangleright$ Time-space shifted local volatility process. We introduce the time-space shifted local volatility process starting from 0 defined for any $x \in \mathbb{R}$ by the following SDE:

$$
\mathrm{d} Z_{t}^{x}=\alpha\left(t, Z_{t}^{x}+x\right) \mathrm{d} W_{t}-\frac{1}{2} \alpha^{2}\left(t, Z_{t}^{x}+x\right) \mathrm{d} t, \quad z_{0}=0 .
$$

$\triangleright$ Integral operators. For any $n \geq 1$, any $l_{1}, . ., l_{n}$ measurable and bounded functions of $t \in\left[0, T+t_{i}\right]$, any $0 \leq s<t \leq T+t_{i}$, we set:

$$
\omega\left(l_{1}, . ., l_{n}\right)_{s}^{t}=\int_{s}^{t} l_{1}\left(r_{1}\right) \int_{r_{1}}^{t} l_{2}\left(r_{2}\right) \ldots \int_{r_{n-1}}^{t} l_{n}\left(r_{n}\right) \mathrm{d} r_{n} \mathrm{~d} r_{n-1} . . \mathrm{d} r_{2} \mathrm{~d} r_{1} .
$$

$\triangleright$ Quadratic mean and total variance. We define the quadratic mean for any bounded measurable function $g$ of $(t, x) \in\left[0, T+t_{i}\right] \times \mathbb{R}$ and for any non empty $[s, t] \subseteq\left[0, T+t_{i}\right]$ at the spatial point $x$ on $[s, t]$ by setting:

$$
\bar{g}_{x}^{s, t}=\sqrt{\frac{1}{t-s} \int_{s}^{t} g^{2}(r, x) \mathrm{d} r .}
$$

For any bounded measurable function $g$ of $(t, x) \in\left[0, T+t_{i}\right] \times \mathbb{R}$ and for any non empty $[s, t] \subseteq\left[0, T+t_{i}\right]$, we define the total variance of $g$ at $x$ on $[s, t]$ as:

$$
\mathcal{V}(g ; x)_{s}^{t}=\int_{s}^{t} g^{2}(r, x) \mathrm{d} r=\left(\bar{g}_{x}^{s, t}\right)^{2}(t-s)
$$

We finally introduce some integral operators $C, \gamma$ and $\pi$ already used in [5]:

Definition 1.1. If the derivatives and the integrals have a meaning, we define for any non empty $[s, t] \subseteq\left[0, T+t_{i}\right]$ and for any bounded measurable function $(l(t, z))_{(t, z) \in\left[0, T+t_{i}\right] \times \mathbb{R}}$ the next operators:

$$
\begin{aligned}
& C_{1}(l ; z)_{s}^{t}=\omega\left(l^{2}(z), l(z) l^{(1)}(z)\right)_{s}^{t}, \quad C_{2}(l ; z)_{s}^{t}=\omega\left(l^{2}(z),\left(l^{(1)}(z)\right)^{2}+l(z) l^{(2)}(z)\right)_{s}^{t}, \\
& C_{3}(l ; z)_{s}^{t}=\omega\left(l^{2}(z), l^{2}(z),\left(l^{(1)}(z)\right)^{2}+l(z) l^{(2)}(z)\right)_{s}^{t}, \quad C_{4}(l ; z)_{s}^{t}=\omega\left(l^{2}(z), l(z) l^{(1)}(z), l(z) l^{(1)}(z)\right)_{s}^{t}, \\
& C_{5}(l ; z)_{s}^{t}=\omega\left(\left(l^{(1)}(z)\right)^{2}+l(z) l^{(2)}(z)\right)_{t}^{s}, \quad C_{6}(l ; z)_{s}^{t}=\omega\left(l(z) l^{(1)}(z), l(z) l^{(1)}(z)\right)_{t}^{s} \text {, } \\
& C_{7}(l ; z)_{s}^{t}=\omega\left(l(z) l^{(1)}(z)\right)_{t}^{s} \text {. }
\end{aligned}
$$

We can define similarly the reverse operators $\widetilde{C}$ obtained by changing the order of integration. For example $\widetilde{C}_{1}(l ; z)_{s}^{t}=\omega\left(l(z) l^{(1)}(z), l^{2}(z)\right)_{s}^{t}$. Supposing in addition that $l$ is non-negative such that $\bar{l}_{z}^{s, t}>0$, we define the 
following operators:

$$
\begin{aligned}
& \gamma_{0}(l ; z)_{s}^{t}=\bar{l}_{z}^{s, t}+\frac{C_{2}(l ; z)_{s}^{t}}{2 \bar{l}_{z}^{s, t}(t-s)}-\frac{C_{4}(l ; z)_{s}^{t}}{4 \bar{l}_{z}^{s, t}(t-s)}-\frac{C_{3}(l ; z)_{s}^{t}}{\left(\bar{l}_{z}^{s, t}\right)^{3}(t-s)^{2}}-\frac{3 C_{4}(l ; z)_{s}^{t}}{\left(\bar{l}_{z}^{s, t}\right)^{3}(t-s)^{2}}+\frac{\left[C_{1}(l ; z)_{s}^{t}\right]^{2}}{8\left(\bar{l}_{z}^{s, t}\right)^{3}(t-s)^{2}}+\frac{3\left[C_{1}(l ; z)_{s}^{t}\right]^{2}}{2\left(\bar{l}_{z}^{s, t}\right)^{5}(t-s)^{3}} \\
& \gamma_{1}(l ; z)_{s}^{t}=\frac{C_{1}(l ; z)_{s}^{t}}{\left(\bar{l}_{z}^{s, t}\right)^{3}(t-s)^{2}}, \quad \gamma_{2}(l ; z)_{s}^{t}=\frac{C_{3}(l ; z)_{s}^{t}}{\left(\bar{l}_{z}^{s, t}\right)^{5}(t-s)^{3}}+3 \frac{C_{4}(l ; z)_{s}^{t}}{\left(\bar{l}_{z}^{s, t}\right)^{5}(t-s)^{3}}-\frac{3\left[C_{1}(l ; z)_{s}^{t}\right]^{2}}{\left(\bar{l}_{z}^{s, t}\right)^{7}(t-s)^{4}} \\
& \pi_{0}(l ; z)_{s}^{t}=\frac{\gamma_{0}(l ; z)_{s}^{t}+\widetilde{\gamma}_{0}(l ; z)_{s}^{t}}{2}, \quad \pi_{1}(l ; z)_{s}^{t}=\frac{\widetilde{\gamma}_{1}(l ; z)_{s}^{t}-\gamma_{1}(l ; z)_{s}^{t}}{2} \\
& \pi_{2}(l ; z)_{s}^{t}=\frac{\gamma_{2}(l ; z)_{s}^{t}+\widetilde{\gamma}_{2}(l ; z)_{s}^{t}}{2}-\frac{C_{5}(l ; z)_{s}^{t}}{8 \bar{l}_{z}^{s, t}(t-s)}+\frac{C_{6}(l ; z)_{s}^{t}}{4\left(\bar{l}_{z}^{s, t}\right)^{3}(t-s)^{2}}
\end{aligned}
$$

where the reverse operators $\widetilde{\gamma}$ are obtained using the reverse operators $\widetilde{C}$.

Remark 1.2. Any of the previously defined operators applied with the function $\alpha$ and the spatial point $x \in \mathbb{R}$ between the dates 0 and $T$ gives the same result as that obtained with $a$ and $x$ between the dates $t_{i}$ and $T+t_{i}$.

$\triangleright$ Forward implied Black-Scholes volatility. For $\left(x_{0}, t_{i}, T, k\right)$ given, the forward implied Black-Scholes volatility of the forward start Call prices is the unique non-negative parameter $\sigma_{\mathrm{I}, \mathrm{F}}\left(x_{0}, t_{i}, T, k\right)$ such that:

$$
\mathrm{Call}^{\mathrm{FS}}\left(e^{x_{0}}, t_{i}, T, e^{k}\right)=\mathrm{Call}^{\mathrm{BS}}\left(0, \sigma_{\mathrm{I}, \mathrm{F}}^{2}\left(x_{0}, t_{i}, T, k\right) T, k\right)
$$

$\triangleright$ New log-strike and new mid-point. We use the notation $k^{\prime}=\frac{k}{2}$ and $x_{\text {avg }}^{\prime}=x_{0}+k^{\prime}=x_{0}+\frac{k}{2}$.

$\triangleright$ About the constants. All our error estimates are stated throughout the paper using the notations:

- " $A=\mathcal{O}(B)$ " means that $|A| \leq C B$ where $C$ stands for a generic constant that is a non-negative increasing function of $T, t_{i}, \mathcal{M}_{0}(a), \mathcal{M}_{1}(a)$ and the oscillation ratio $\frac{1}{\mathcal{C}_{a}}$.

- Similarly, if $A$ is non-negative, $A \leq_{c} B$ means that $A \leq C B$ for a generic constant $C$.

We finally announce a technical result related to the derivatives of Call ${ }^{\mathrm{BS}}$ w.r.t. the volatility parameter.

Lemma 1.3. Let $x, k \in \mathbb{R}, \nu>0$ and $T>0$. We introduce $\operatorname{Vega}^{\mathrm{BS}}\left(x, \nu^{2} T, k\right)=\partial_{\nu} \mathrm{Call}^{\mathrm{BS}}\left(x, \nu^{2} T, k\right)$, $\operatorname{Vomma}^{\mathrm{BS}}\left(x, \nu^{2} T, k\right)=\partial_{\nu^{2}}^{2} \mathrm{Call}^{\mathrm{BS}}\left(x, \nu^{2} T, k\right)$ and $\operatorname{Ultima}^{\mathrm{BS}}\left(x, \nu^{2} T, k\right)=\partial_{\nu^{3}}^{3} \mathrm{Call}^{\mathrm{BS}}\left(x, \nu^{2} T, k\right)$. For any integer $m \geq 0$, we have for a generic constant depending polynomially on $\nu \sqrt{T}$ :

$$
\begin{aligned}
|x-k|^{m}\left|\operatorname{Vega}^{\mathrm{BS}}\left(x, \nu^{2} T, k\right)\right| & \leq_{c} \sqrt{T}(\nu \sqrt{T})^{m}, \quad|x-k|^{m}\left|\operatorname{Vomma}^{\mathrm{BS}}\left(x, \nu^{2} T, k\right)\right| \leq_{c} T(\nu \sqrt{T})^{m-1}, \\
|x-k|^{m}\left|\operatorname{Ultima}^{\mathrm{BS}}\left(x, \nu^{2} T, k\right)\right| & \leq_{c} T^{\frac{3}{2}}(\nu \sqrt{T})^{m-2},
\end{aligned}
$$

Proof. For the first inequality, apply [5, Proposition 34] to write that $\operatorname{Vega~}^{\mathrm{BS}}\left(x, \nu^{2} T, k\right)=\nu T\left(\partial_{x^{2}}^{2}-\partial_{x}\right) \mathrm{Call}^{\mathrm{BS}}\left(x, \nu^{2} T, k\right)$ [5, Corollary 30]. For the second use [5, Proposition 32] to write that:

$$
\operatorname{Vomma}^{\mathrm{BS}}\left(x, \nu^{2} T, k\right)=\frac{\operatorname{Vega}^{\mathrm{BS}}\left(x, \nu^{2} T, k\right)}{\nu}\left[\frac{(x-k)^{2}}{\nu^{2} T}-\frac{\nu^{2} T}{4}\right]
$$

and conclude again with [5, Proposition 34 and Corollary 30]. The last inequality is handled similarly using [5, Propositions 32, 34 and Corollary 30].

\section{THIRD ORDER FORWARD IMPLIED VOLATILITY EXPANSION}

We announce the main result of the paper: 
Theorem 2.1. (3rd order expansion of the forward implied volatility). Assume $\left(\mathcal{H}_{a}\right)$ and let a fixed small constant $\xi>0$. Then suppose that $\mathcal{M}_{1}(a), \mathcal{M}_{0}(a), T, t_{i}$ and $|k|$ are globally small enough to ensure that:

$$
\begin{aligned}
\pi^{k}(\alpha ; x)_{0}^{T}=\pi^{k}(a ; x)_{t_{i}}^{t_{i}+T} & =\pi_{0}(\alpha ; x)_{0}^{T}-k \pi_{1}(\alpha ; x)_{0}^{T}+k^{2} \pi_{2}(\alpha ; x)_{0}^{T}>\xi \mathcal{M}_{0}(a)>0, \quad \forall x \in \mathbb{R}, \\
\tilde{\sigma}_{\mathrm{I}, \mathrm{F}}^{3, x_{\text {avg }}^{\prime}}\left(x_{0}, t_{i}, T, k\right) & =\pi^{k}\left(a ; x_{\text {avg }}^{\prime}\right)_{t_{i}}^{T+t_{i}}+\pi_{\mathrm{F}}^{k}\left(a ; x_{\text {avg }}^{\prime}\right)_{t_{i}}^{T+t_{i}}>\xi \mathcal{M}_{0}(a)>0,
\end{aligned}
$$

where the operators $\pi_{0}, \pi_{1}, \pi_{2}$ are defined in Definition 1.1, where:

$$
\begin{aligned}
& \pi_{\mathrm{F}}^{k}\left(a ; x_{a v g}^{\prime}\right)_{t_{i}}^{T+t_{i}}=\frac{C_{7}\left(a ; x_{a v g}^{\prime}\right)_{t_{i}}^{T+t_{i}}}{2 \bar{a}_{x_{a v g}^{\prime}, T+t_{i}}^{t_{i}} T}\left[-\mathcal{V}\left(a ; x_{0}\right)_{0}^{t_{i}}+C_{1}\left(a ; x_{0}\right)_{0}^{t_{i}}\right]+\left[\frac{C_{5}\left(a ; x_{a v g}^{\prime}\right)_{t_{i}}^{T+t_{i}}}{2 \bar{a}_{x_{a v g}}^{t_{i}, T+t_{i}} T}-\frac{C_{6}\left(a ; x_{a v g}^{\prime}\right)_{t_{i}}^{T+t_{i}}}{\left(\bar{a}_{x_{a v g}}^{t_{i}, T+t_{i}}\right)^{3} T^{2}}\right] \\
& \times\left[\mathcal{V}\left(a ; x_{0}\right)_{0}^{t_{i}}+\frac{1}{4} \mathcal{V}^{2}\left(a ; x_{0}\right)_{0}^{t_{i}}\right]+\left[\frac{k^{2}}{\left(\pi^{k}\left(a ; x_{a v g}^{\prime}\right)_{t_{i}}^{T+t_{i}}\right)^{2} T}-\frac{\left(\pi^{k}\left(a ; x_{a v g}^{\prime}\right)_{t_{i}}^{T+t_{i}}\right)^{2} T}{4}\right] \frac{C_{6}\left(a ; x_{a v g}^{\prime}\right)_{t_{i}}^{T+t_{i}}}{\left(\bar{a}_{x_{a v g}^{\prime}}^{t_{i}, T+t_{i}}\right)^{2} \pi^{k}\left(a ; x_{a v g}^{\prime}\right)_{t_{i}}^{T+t_{i}} T^{2}} \mathcal{V}\left(a ; x_{0}\right)_{0}^{t_{i}} \\
& +\frac{k}{2}\left[\frac{C_{2}\left(\tilde{a} ; x_{a v g}^{\prime}\right)_{t_{i}}^{T+t_{i}}-C_{2}\left(a ; x_{a v g}^{\prime}\right)_{t_{i}}^{T+t_{i}}}{2\left(\bar{a}_{x}^{t_{i}, T+t_{i}}\right)^{3} T^{2}}+3 \frac{C_{7}\left(a ; x_{a v g}\right)_{t_{i}}^{T+t_{i}}\left(C_{1}\left(a ; x_{a v g}^{\prime}\right)_{t_{i}}^{T+t_{i}}-C_{1}\left(\tilde{a} ; x_{a v g}^{\prime}\right)_{t_{i}}^{T+t_{i}}\right)}{2\left(\bar{a}_{x}^{t_{i}, T+t_{i}}\right)^{5} T^{3}}\right] \mathcal{V}\left(a ; x_{0}\right)_{t_{i}}^{T+t_{i}}
\end{aligned}
$$

and where the operators $C_{1}, C_{2}, C_{5}, C_{6}$ and $C_{7}$ are defined in Definition 1.1. Then $\tilde{\sigma}_{\mathrm{I}, \mathrm{F}}^{3, x_{\text {avg }}^{\prime}}\left(x_{0}, t_{i}, T, k\right)$ is a third order approximation of $\sigma_{\mathrm{I}, \mathrm{F}}\left(x_{0}, t_{i}, T, k\right)$ in the following sense:

$$
\operatorname{Call}^{\mathrm{FS}}\left(e^{x_{0}}, t_{i}, T, e^{k}\right)=\operatorname{Call}^{\mathrm{BS}}\left(0,\left(\tilde{\sigma}_{\mathrm{I}, \mathrm{F}}^{3, x_{\text {avg }}^{\prime}}\left(x_{0}, t_{i}, T, k\right)\right)^{2} T, k\right)+\mathcal{O}\left(\mathcal{M}_{1}(a)\left[\mathcal{M}_{0}(a)\right]^{3} \sqrt{T}\left(\sqrt{t_{i}}+\sqrt{T}\right)^{3}\right),
$$

where the constant in the above estimate notably depends on the oscillation ratio $\frac{1}{\xi}$.

If a is time-independent, using Definition 1.1, $\pi^{k}(a ; x)_{t_{i}}^{t_{i}+T}$ and $\pi_{\mathrm{F}}^{k}(a ; x)_{t_{i}}^{t_{i}+T}$ are explicitly given by:

$$
\begin{aligned}
\pi^{k}(a ; x)_{t_{i}}^{t_{i}+T} & =a(x)\left\{1+T\left[\frac{a(x) a^{(2)}(x)}{12}-\left(a^{(1)}\right)^{2}(x)\left(\frac{1}{24}+\frac{a^{2}(x) T}{96}\right)\right]+k^{2}\left[\frac{a^{(2)}(x)}{24 a(x)}-\frac{\left(a^{(1)}\right)^{2}(x)}{12 a^{2}(x)}\right]\right\}, \\
\pi_{\mathrm{F}}^{k}\left(a ; x_{\text {avg }}^{\prime}\right)_{t_{i}}^{T+t_{i}} & =\frac{a^{2}\left(x_{0}\right) t_{i}}{2}\left\{a^{(1)}\left(x_{a v g}^{\prime}\right)\left[-1+\frac{a\left(x_{0}\right) a^{(1)}\left(x_{0}\right) t_{i}}{2}\right]+a^{(2)}\left(x_{a v g}^{\prime}\right)\left[1+\frac{a^{2}\left(x_{0}\right) t_{i}}{4}\right]\right. \\
& \left.+\frac{\left(a^{(1)}\left(x_{a v g}^{\prime}\right)\right)^{2}}{\pi^{k}\left(a ; x_{\text {avg }}^{\prime}\right)_{t_{i}}^{T+t_{i}}}\left[\frac{k^{2}}{\left(\pi^{k}\left(a ; x_{\text {avg }}^{\prime}\right)_{t_{i}}^{T+t_{i}}\right)^{2} T}-\frac{\left(\pi^{k}\left(a ; x_{a v g}^{\prime}\right)_{t_{i}}^{T+t_{i}}\right)^{2} T}{4}\right]\right\} .
\end{aligned}
$$

Remark 2.2. If $t_{i}=0, \pi_{\mathrm{F}}^{k}\left(a ; x_{a v g}^{\prime}\right)_{t_{i}}^{T+t_{i}}$ vanishes and the above approximation reduces to $\pi^{k}\left(a ; x_{\text {avg }}^{\prime}\right)_{t_{i}}^{T+t_{i}}$ which is the approximation of the vanilla third order implied volatility expansion given in [5, Theorem 22]. The additional term $\pi_{\mathrm{F}}^{k}\left(a ; x_{a v g}^{\prime}\right)_{t_{i}}^{T+t_{i}}$ due to the forward start is therefore interpreted as a forward bias.

Remark 2.3. If one prefers to restrict to a second order approximation, it simply writes:

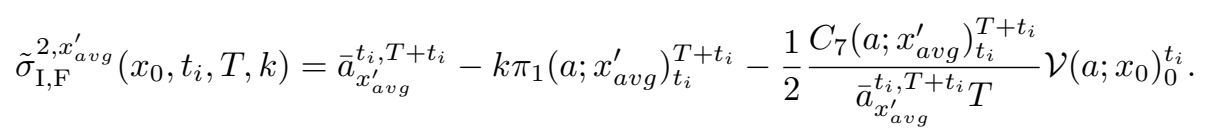

We let the reader verify in view of the additional terms in (4), of (5) and of Lemma 1.3 that $\operatorname{Call}^{\mathrm{FS}}\left(e^{x_{0}}, t_{i}, T, e^{k}\right)=\mathrm{Call}^{\mathrm{BS}}\left(0,\left(\tilde{\sigma}_{\mathrm{I}, \mathrm{F}}^{2, x^{\prime}}{ }^{\prime}\left(x_{0}, t_{i}, T, k\right)\right)^{2} T, k\right)+\mathcal{O}\left(\mathcal{M}_{1}(a)\left[\mathcal{M}_{0}(a)\right]^{2} \sqrt{T}\left(\sqrt{t_{i}}+\sqrt{T}\right)^{2}\right)$.

Proof. We use the Markov property of the processes $X$ to get:

$$
\text { Call }^{\mathrm{FS}}\left(e^{x_{0}}, t_{i}, T, e^{k}\right)=\mathbb{E}\left[\mathbb{E}\left[\left(e^{X_{t_{i}+T}-X_{t_{i}}}-e^{k}\right)_{+} \mid X_{t_{i}}\right]\right] .
$$


Then using the deterministic time change $t \rightarrow t+t_{i}$ for any $t \in[0, T]$ and [15, Propositions 5.1.4 and 5.1.5], we easily see that under the conditional knowledge of $X_{t_{i}}, X_{t_{i}+T}-X_{t_{i}}$ has the same law that $Z_{T}^{X_{t_{i}}}$ where $\left(Z_{t}^{x}\right)_{t \in[0, T]}$ is the solution of the $\operatorname{SDE}(2)$. Thus we have $\mathbb{E}\left[\left(e^{X_{t_{i}+T}-X_{t_{i}}}-e^{k}\right)_{+} \mid X_{t_{i}}\right]=\mathbb{E}\left[\left(e^{Z_{T}^{X_{t_{i}}}}-e^{k}\right)_{+} \mid X_{t_{i}}\right]$. Next remark that $\mathbb{E}\left[\left(e^{Z_{T}^{X_{t_{i}}}}-e^{k}\right)_{+} \mid X_{t_{i}}\right]$ is a Call option price at time 0 with maturity $T$, strike $e^{k}$, spot 1 written on the log-asset $Z_{T}^{X_{t_{i}}}$ with local volatility function $(t, x) \rightarrow \alpha\left(t, x+X_{t_{i}}\right)$. Then owing to the assumed positivity of $\pi^{k}(\alpha ; x)_{0}^{T}, \forall x \in \mathbb{R}$, we can follow the proof of [5, Theorem 22] and one easily obtains with Lemma 1.3:

$$
\mathbb{E}\left[\left(e^{Z_{T}^{X_{t_{i}}}}-e^{k}\right)_{+} \mid X_{t_{i}}\right]=\operatorname{Call}^{\mathrm{BS}}\left(0,\left(\pi^{k}\left(\alpha ; k^{\prime}+X_{t_{i}}\right)_{0}^{T}\right)^{2} T, k\right)+\operatorname{Error}_{3, k^{\prime}}\left(X_{t_{i}}\right),
$$

where Error $_{3, k^{\prime}}\left(X_{t_{i}}\right)$ depends on $X_{t_{i}}$ only throughout the function $a$ (as a shift parameter) and its derivatives which are bounded functions. In addition we have a.s. $\left|\operatorname{Error}_{3, k^{\prime}}\left(X_{t_{i}}\right)\right| \leq_{c} \mathcal{M}_{1}(a)\left[\mathcal{M}_{0}(a)\right]^{3} T^{2}$. Then using $\pi^{k}\left(\alpha ; k^{\prime}+X_{t_{i}}\right)_{0}^{T}=\pi^{k}\left(a ; k^{\prime}+X_{t_{i}}\right)_{t_{i}}^{T+t_{i}}$ (see remark 1.2), the price (8) can be finally written as:

$$
\operatorname{Call}^{\mathrm{FS}}\left(e^{x_{0}}, t_{i}, T, e^{k}\right)=\mathbb{E}\left[\mathrm{Call}^{\mathrm{BS}}\left(0,\left(\pi^{k}\left(a ; k^{\prime}+X_{t_{i}}\right)_{t_{i}}^{T+t_{i}}\right)^{2} T, k\right)\right]+\mathcal{O}\left(\mathcal{M}_{1}(a)\left[\mathcal{M}_{0}(a)\right]^{3} T^{2}\right)
$$

Now apply a Taylor expansion for the smooth function $\nu \rightarrow$ Call $^{\mathrm{BS}}\left(0, \nu^{2} T, k\right)$ at $\nu=\pi^{k}\left(a ; k^{\prime}+X_{t_{i}}\right)_{t_{i}}^{T+t_{i}}$ around $\nu=\pi^{k}\left(a ; x_{a v g}^{\prime}\right)_{t_{i}}^{T+t_{i}}$

$$
\begin{aligned}
\mathbb{E}\left[\operatorname{Call}^{\mathrm{BS}}(0,\right. & \left.\left.\left(\pi^{k}\left(a ; k^{\prime}+X_{t_{i}}\right)_{t_{i}}^{T+t_{i}}\right)^{2} T, k\right)\right]=\operatorname{Call}^{\mathrm{BS}}\left(0,\left(\pi^{k}\left(a ; x_{a v g}^{\prime}\right)_{t_{i}}^{T+t_{i}}\right)^{2} T, k\right) \\
& +\operatorname{Vega}^{\mathrm{BS}}\left(0,\left(\pi^{k}\left(a ; x_{a v g}^{\prime}\right)_{t_{i}}^{T+t_{i}}\right)^{2} T, k\right) \mathbb{E}\left[\pi^{k}\left(a ; k^{\prime}+X_{t_{i}}\right)_{t_{i}}^{T+t_{i}}-\pi^{k}\left(a ; x_{a v g}^{\prime}\right)_{t_{i}}^{T+t_{i}}\right] \\
& +\frac{1}{2} \operatorname{Vomma}^{\mathrm{BS}}\left(0,\left(\pi^{k}\left(a ; x_{a v g}^{\prime}\right)_{t_{i}}^{T+t_{i}}\right)^{2} T, k\right) \mathbb{E}\left[\left(\pi^{k}\left(a ; k^{\prime}+X_{t_{i}}\right)_{t_{i}}^{T+t_{i}}-\pi^{k}\left(a ; x_{a v g}^{\prime}\right)_{t_{i}}^{T+t_{i}}\right)^{2}\right] \\
& +R
\end{aligned}
$$

where Vega ${ }^{\mathrm{BS}}$, Vomma ${ }^{\mathrm{BS}}$ and Ultima $^{\mathrm{BS}}$ are defined in Lemma 1.3 and where:

$$
\begin{aligned}
R= & \mathbb{E}\left[\left(\pi^{k}\left(a ; k^{\prime}+X_{t_{i}}\right)_{t_{i}}^{T+t_{i}}-\pi^{k}\left(a ; x_{a v g}^{\prime}\right)_{t_{i}}^{T+t_{i}}\right)^{3}\right. \\
& \left.\times\left.\int_{0}^{1} \operatorname{Ultima}^{\mathrm{BS}}\left(0, \nu^{2} T, k\right)\right|_{\nu=(1-\lambda) \pi^{k}\left(a ; x_{a v g}^{\prime}\right)_{t_{i}}^{T+t_{i}}+\lambda \pi^{k}\left(a ; k^{\prime}+X_{t_{i}}\right)_{t_{i}}^{T+t_{i}}} \frac{(1-\lambda)^{2}}{2} \mathrm{~d} \lambda\right] .
\end{aligned}
$$

First notice that we readily have using Lemma 1.3 and the ellipticity assumptions:

$$
R=\mathcal{O}\left(\mathcal{M}_{1}(a)\left[\mathcal{M}_{0}(a)\right]^{3} t_{i}^{\frac{3}{2}} \sqrt{T}\right)
$$

Then we expand the functions $x \rightarrow \bar{a}_{x}^{t_{i}, T+t_{i}}, x \rightarrow \pi_{0}(a ; x)_{t_{i}}^{T+t_{i}}-\bar{a}_{x}^{t_{i}, T+t_{i}}$ and $x \rightarrow \pi_{i}(a ; x)_{t_{i}}^{T+t_{i}}, i \in\{1,2\}$.

$\triangleright$ Step 1: Expansion of $\bar{a}_{x}^{t_{i}, T+t_{i}}$. We aim at showing that:

$$
\begin{aligned}
& \operatorname{Vega}^{\mathrm{BS}}\left(0,\left(\pi^{k}\left(a ; x_{a v g}^{\prime}\right)_{t_{i}}^{T+t_{i}}\right)^{2} T, k\right) \mathbb{E}\left[\bar{a}_{k^{\prime}+X_{t_{i}}}^{t_{i}, T+t_{i}}-\bar{a}_{x_{a v g}^{\prime}}^{t_{i}, T+t_{i}}\right] \\
= & \operatorname{Vega}^{\mathrm{BS}}\left(0,\left(\pi^{k}\left(a ; x_{a v g}^{\prime}\right)_{t_{i}}^{T+t_{i}}\right)^{2} T, k\right)\left\{\frac{C_{7}\left(a ; x_{a v g}^{\prime}\right)_{t_{i}}^{T+t_{i}}}{2 \bar{a}_{x_{a v g}^{\prime}, T+t_{i}}^{t_{i}} T}\left[-\mathcal{V}\left(a ; x_{0}\right)_{0}^{t_{i}}+C_{1}\left(a ; x_{0}\right)_{0}^{t_{i}}\right]\right. \\
& \left.+\left[\frac{C_{5}\left(a ; x_{a v g}^{\prime}\right)_{t_{i}}^{T+t_{i}}}{2 \bar{a}_{x_{a v g}^{\prime}}^{t_{i}, T+t_{i}} T}-\frac{C_{6}\left(a ; x_{a v g}^{\prime}\right)_{t_{i}}^{T+t_{i}}}{\left(\bar{a}_{x_{a v g}^{\prime}, T+t_{i}}^{t_{i}}\right)^{3} T^{2}}\right]\left[\mathcal{V}\left(a ; x_{0}\right)_{0}^{t_{i}}+\frac{1}{4} \mathcal{V}^{2}\left(a ; x_{0}\right)_{0}^{t_{i}}\right]\right\}+\mathcal{O}\left(\mathcal{M}_{1}(a)\left[\mathcal{M}_{0}(a)\right]^{3} t_{i}^{\frac{3}{2}} \sqrt{T}\right),
\end{aligned}
$$




$$
\begin{aligned}
& \frac{1}{2} \operatorname{Vomma}^{\mathrm{BS}}\left(0,\left(\pi^{k}\left(a ; x_{a v g}^{\prime}\right)_{t_{i}}^{T+t_{i}}\right)^{2} T, k\right) \mathbb{E}\left[\left(\bar{a}_{k^{\prime}+X_{t_{i}}}^{t_{i}, T+t_{i}}-\bar{a}_{x_{a v g}^{\prime}}^{t_{i}, T+t_{i}}\right)^{2}\right] \\
= & \frac{1}{2} \operatorname{Vomma}^{\mathrm{BS}}\left(0,\left(\pi^{k}\left(a ; x_{a v g}^{\prime}\right)_{t_{i}}^{T+t_{i}}\right)^{2} T, k\right)\left\{\left(\frac{C_{7}\left(a ; x_{a v g}^{\prime}\right)_{t_{i}}^{T+t_{i}}}{2 \bar{a}_{x_{a v g}^{\prime}, T+t_{i}}^{t_{i}} T} \mathcal{V}\left(a ; x_{0}\right)_{0}^{t_{i}}\right)^{2}+2 \frac{C_{6}\left(a ; x_{a v g}^{\prime}\right)_{t_{i}}^{T+t_{i}}}{\left(\bar{a}_{x_{a v g}^{\prime}}^{t_{i}, T+t_{i}}\right)^{2} T^{2}} \mathcal{V}\left(a ; x_{0}\right)_{0}^{t_{i}}\right\} \\
& +\mathcal{O}\left(\mathcal{M}_{1}(a)\left[\mathcal{M}_{0}(a)\right]^{3} t_{i}^{\frac{3}{2}} \sqrt{T}\right) .
\end{aligned}
$$

We begin with the proof of (12). Expand $\bar{a}_{x}^{t_{i}, T+t_{i}}$ to write:

$$
\begin{aligned}
& \mathbb{E}\left[\bar{a}_{k^{\prime}+X_{t_{i}}}^{t_{i}, T+t_{i}}-\bar{a}_{x_{a v g}^{\prime}, T+t_{i}}^{t_{i}, T}=\frac{C_{7}\left(a ; x_{a v g}^{\prime}\right)_{t_{i}}^{T+t_{i}}}{\bar{a}_{x_{a v g}^{\prime}, T+t_{i}}^{\prime} T} \mathbb{E}\left[\left(X_{t_{i}}-x_{0}\right)\right]+\left.\frac{1}{2} \partial_{x^{2}}^{2} \bar{a}_{x}^{t_{i}, T+t_{i}}\right|_{x=x_{a v g}^{\prime}} \mathbb{E}\left[\left(X_{t_{i}}-x_{0}\right)^{2}\right]+R_{1},\right. \\
& \left.\partial_{x^{2}}^{2} \bar{a}_{x}^{t_{i}, T+t_{i}}\right|_{x=x_{a v g}^{\prime}}=\frac{C_{5}\left(a ; x_{a v g}^{\prime}\right)_{t_{i}}^{T+t_{i}}}{\bar{a}_{x_{a v g}^{t_{i}, T+t_{i}} T}^{\prime} T}-\frac{\left[C_{7}\left(a ; x_{a v g}^{\prime}\right)_{t_{i}}^{T+t_{i}}\right]^{2}}{\left(\bar{a}_{x_{a v g}^{\prime}, T+t_{i}}^{\prime}\right)^{3} T^{2}}=\frac{C_{5}\left(a ; x_{a v g}^{\prime}\right)_{t_{i}}^{T+t_{i}}}{\bar{a}_{x_{a v g}^{\prime}}^{t_{i} T+t_{i}} T}-2 \frac{C_{6}\left(a ; x_{a v g}^{\prime}\right)_{t_{i}}^{T+t_{i}}}{\left(\bar{a}_{x_{a v g}}^{t_{i}, T+t_{i}}\right)^{3} T^{2}}, \\
& R_{1}=\mathbb{E}\left[\left.\left(X_{t_{i}}-x_{0}\right)^{3} \int_{0}^{1} \partial_{x^{3}}^{3} \bar{a}_{x}^{t_{i}, T+t_{i}}\right|_{x=k^{\prime}+\lambda X_{t_{i}}+(1-\lambda) x_{0}} \frac{(1-\lambda)^{2}}{2} \mathrm{~d} \lambda\right],
\end{aligned}
$$

where the operators $C_{5}, C_{6}$ and $C_{7}$ are defined in Definition 1.1. We readily have using Lemma 1.3:

$$
R_{1} \operatorname{Vega}^{\mathrm{BS}}\left(0,\left(\pi^{k}\left(a ; x_{\text {avg }}^{\prime}\right)_{t_{i}}^{T+t_{i}}\right)^{2} T, k\right)=\mathcal{O}\left(\mathcal{M}_{1}(a)\left[\mathcal{M}_{0}(a)\right]^{3} t_{i}^{\frac{3}{2}} \sqrt{T}\right)
$$

Then we introduce the corrective processes $\left(X_{1, t}\right)_{t \in\left[0, T+t_{i}\right]}-\left(X_{2, t}\right)_{t \in\left[0, T+t_{i}\right]}$ defined by:

$$
\begin{aligned}
& \mathrm{d} X_{1, t}=a\left(t, x_{0}\right)\left(\mathrm{d} W_{t}-\frac{1}{2} a\left(t, x_{0}\right) \mathrm{d} t\right), X_{1,0}=0, \\
& \mathrm{~d} X_{2, t}=2 a^{(1)}\left(t, x_{0}\right) X_{1, t}\left(\mathrm{~d} W_{t}-a\left(t, x_{0}\right) \mathrm{d} t\right), X_{2,0}=0 .
\end{aligned}
$$

Using the fact that the process $\left(\int_{0}^{t} a^{(1)}\left(s, x_{0}\right) X_{1, s} \mathrm{~d} W_{s}\right)_{t \in\left[0, T+t_{i}\right]}$ is a martingale and the $\mathrm{L}^{p}$ upper bounds for any $p \geq 1$ of the corrective processes (see [1]), we obtain the following weak approximations:

$$
\begin{aligned}
\mathbb{E}\left[X_{t_{i}}-x_{0}\right] & =\mathbb{E}\left[X_{1, t_{i}}\right]+\mathcal{O}\left(\mathcal{M}_{1}(a)\left[\mathcal{M}_{0}(a)\right] t_{i}\right)=\mathbb{E}\left[X_{1, t_{i}}\right]+\frac{1}{2} \mathbb{E}\left[X_{2, t_{i}}\right]+\mathcal{O}\left(\mathcal{M}_{1}(a)\left[\mathcal{M}_{0}(a)\right]^{2} t_{i}^{\frac{3}{2}}\right) \\
& =-\frac{1}{2} \mathcal{V}\left(a ; x_{0}\right)_{0}^{t_{i}}+\mathcal{O}\left(\mathcal{M}_{1}(a)\left[\mathcal{M}_{0}(a)\right] t_{i}\right)=-\frac{1}{2} \mathcal{V}\left(a ; x_{0}\right)_{0}^{t_{i}}+\frac{1}{2} C_{1}\left(a ; x_{0}\right)_{0}^{t_{i}}+\mathcal{O}\left(\mathcal{M}_{1}(a)\left[\mathcal{M}_{0}(a)\right]^{2} t_{i}^{\frac{3}{2}}\right), \\
\mathbb{E}\left[\left(X_{t_{i}}-x_{0}\right)^{2}\right] & =\mathbb{E}\left[X_{1, t_{i}}^{2}\right]+\mathcal{O}\left(\mathcal{M}_{1}(a)\left[\mathcal{M}_{0}(a)\right]^{2} t_{i}^{\frac{3}{2}}\right)=\frac{1}{4} \mathcal{V}^{2}\left(a ; x_{0}\right)_{0}^{t_{i}}+\mathcal{V}\left(a ; x_{0}\right)_{0}^{t_{i}}+\mathcal{O}\left(\mathcal{M}_{1}(a)\left[\mathcal{M}_{0}(a)\right]^{2} t_{i}^{\frac{3}{2}}\right) .
\end{aligned}
$$

Combining (14)-(15)-(16)-(17) and Lemma 1.3 leads to the announced result. For (13) write using (17):

$$
\begin{aligned}
& \mathbb{E}\left[\left(\bar{a}_{k^{\prime}+X_{t_{i}}}^{t_{i}, T+t_{i}}-\bar{a}_{x_{a v g}^{\prime}}^{t_{i}, T+t_{i}}\right)^{2}\right]=\left(\frac{C_{7}\left(a ; x_{\text {avg }}^{\prime}\right)_{t_{i}}^{T+t_{i}}}{\bar{a}_{x_{a v g}^{\prime}, T+t_{i}}^{t^{\prime}} T}\right)^{2} \mathbb{E}\left[\left(X_{t_{i}}-x_{0}\right)^{2}\right]+R_{2}, \\
& =\left(\frac{C_{7}\left(a ; x_{a v g}^{\prime}\right)_{t_{i}}^{T+t_{i}}}{\bar{a}_{x_{\text {avg }}^{t_{i}, T+t_{i}} T}^{\prime} T}\right)^{2}\left(\frac{1}{4} \mathcal{V}^{2}\left(a ; x_{0}\right)_{0}^{t_{i}}+\mathcal{V}\left(a ; x_{0}\right)_{0}^{t_{i}}\right)+\mathcal{O}\left(\mathcal{M}_{1}(a)\left[\mathcal{M}_{0}(a)\right]^{4} t_{i}^{\frac{3}{2}}\right)+R_{2}
\end{aligned}
$$

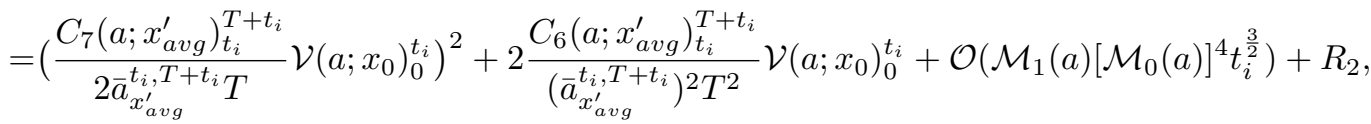


where setting $R_{3}=\left.\left(X_{t_{i}}-x_{0}\right)^{2} \int_{0}^{1} \partial_{x^{2}}^{2} \bar{a}_{x}^{t_{i}, T+t_{i}}\right|_{x=k^{\prime}+\lambda X_{t_{i}}+(1-\lambda) x_{0}}(1-\lambda) \mathrm{d} \lambda$, $R_{2}=\mathbb{E}\left[R_{3}^{2}+2 R_{3} \frac{C_{7}\left(a ; x_{a v g}^{\prime}\right)_{t_{i}}^{T+t_{i}}}{\bar{a}_{x_{a v g}^{\prime}, T+t_{i}}^{\prime} T}\left(X_{t_{i}}-x_{0}\right)\right]=\mathcal{O}\left(\mathcal{M}_{1}(a)\left[\mathcal{M}_{0}(a)\right]^{4} t_{i}^{\frac{3}{2}}\right)$. We Achieve the proof with Lemma 1.3.

$\triangleright$ Step 2: Expansion of $\pi_{1}(a ; x)_{t_{i}}^{T+t_{i}}$. We now prove the following result for $\pi_{1}(a ; x)_{t_{i}}^{T+t_{i}}$ :

$$
\begin{aligned}
& \operatorname{Vega}^{\mathrm{BS}}\left(0,\left(\pi^{k}\left(a ; x_{a v g}^{\prime}\right)_{t_{i}}^{T+t_{i}}\right)^{2} T, k\right) k \mathbb{E}\left[\pi_{1}\left(a ; x_{a v g}^{\prime}\right)_{t_{i}}^{T+t_{i}}-\pi_{1}\left(a ; k^{\prime}+X_{t_{i}}\right)_{t_{i}}^{T+t_{i}}\right] \\
= & \operatorname{Vega}^{\mathrm{BS}}\left(0,\left(\pi^{k}\left(a ; x_{a v g}^{\prime}\right)_{t_{i}}^{T+t_{i}}\right)^{2} T, k\right) \frac{k}{2} \mathcal{V}\left(a ; x_{0}\right)_{t_{i}}^{T+t_{i}}\left[\frac{C_{2}\left(\tilde{a} ; x_{a v g}^{\prime}\right)_{t_{i}}^{T+t_{i}}-C_{2}\left(a ; x_{a v g}^{\prime}\right)_{t_{i}}^{T+t_{i}}}{2\left(\bar{a}_{x}^{t_{i}, T+t_{i}}\right)^{3} T^{2}}\right. \\
& \left.+3 \frac{C_{7}\left(a ; x_{a v g}^{\prime}\right)_{t_{i}}^{T+t_{i}}\left(C_{1}\left(a ; x_{a v g}^{\prime}\right)_{t_{i}}^{T+t_{i}}-C_{1}\left(\tilde{a} ; x_{a v g}^{\prime}\right)_{t_{i}}^{T+t_{i}}\right)}{2\left(\bar{a}_{x}^{t_{i}, T+t_{i}}\right)^{5} T^{3}}\right]+\mathcal{O}\left(\mathcal{M}_{1}(a)\left[\mathcal{M}_{0}(a)\right]^{3} T t_{i}\right) .
\end{aligned}
$$

We have using the definition of $\pi_{1}$ :

$$
\begin{aligned}
& \mathbb{E}\left[\pi_{1}\left(a ; k^{\prime}+X_{t_{i}}\right)_{t_{i}}^{T+t_{i}}-\pi_{1}\left(a ; x_{a v g}^{\prime}\right)_{t_{i}}^{T+t_{i}}\right]=\left.\partial_{x} \pi_{1}(a ; x)_{t_{i}}^{T+t_{i}}\right|_{x=x_{a v g}^{\prime}} \mathbb{E}\left[X_{t_{i}}-x_{0}\right]+R_{4} \\
& \partial_{x} \pi_{1}(a ; x)_{t_{i}}^{T+t_{i}}=\frac{1}{2}\left(\partial_{x} \frac{C_{1}(\tilde{a} ; x)_{t_{i}}^{T+t_{i}}}{\left(\bar{a}_{x}^{t_{i}, T+t_{i}}\right)^{3} T^{2}}-\partial_{x} \frac{C_{1}(a ; x)_{t_{i}}^{T+t_{i}}}{\left(\bar{a}_{x}^{t_{i}, T+t_{i}}\right)^{3} T^{2}}\right) \\
& \partial_{x} \frac{C_{1}(a ; x)_{t_{i}}^{T+t_{i}}}{\left(\bar{a}_{x}^{t_{i}, T+t_{i}}\right)^{3} T^{2}}=\frac{C_{2}(a ; x)_{t_{i}}^{T+t_{i}}+2 C_{6}(a ; x)_{t_{i}}^{T+t_{i}}}{\left(\bar{a}_{x}^{t_{i}, T+t_{i}}\right)^{3} T^{2}}-3 \frac{C_{7}(a ; x)_{t_{i}}^{T+t_{i}} C_{1}(a ; x)_{t_{i}}^{T+t_{i}}}{\left(\bar{a}_{x}^{t_{i}, T+t_{i}}\right)^{5} T^{3}} \\
& R_{4}=\mathbb{E}\left[\left(X_{t_{i}}-x_{0}\right)^{2} \times\left.\int_{0}^{1} \partial_{x^{2}}^{2} \pi_{1}(a ; x)_{t_{i}}^{T+t_{i}}\right|_{x=k^{\prime}+\lambda X_{t_{i}}+(1-\lambda) x_{0}}(1-\lambda) \mathrm{d} \lambda\right]=\mathcal{O}\left(\mathcal{M}_{1}(a)\left[\mathcal{M}_{0}(a)\right]^{2} t_{i}\right)
\end{aligned}
$$

Hence using (16)-(20)-(21)-(22) and the symmetry of the operator $C_{6}$, we get for (19):

$$
\begin{aligned}
& \mathbb{E}\left[\pi_{1}\left(a ; k^{\prime}+X_{t_{i}}\right)_{t_{i}}^{T+t_{i}}-\pi_{1}\left(a ; x_{a v g}^{\prime}\right)_{t_{i}}^{T+t_{i}}\right] \\
= & -\frac{1}{2} \mathcal{V}\left(a ; x_{0}\right)_{t_{i}}^{T+t_{i}}\left[\frac{C_{2}\left(\tilde{a} ; x_{a v g}^{\prime}\right)_{t_{i}}^{T+t_{i}}-C_{2}\left(a ; x_{a v g}^{\prime}\right)_{t_{i}}^{T+t_{i}}}{2\left(\bar{a}_{x}^{t_{i}, T+t_{i}}\right)^{3} T^{2}}+3 \frac{C_{7}\left(a ; x_{a v g}^{\prime}\right)_{t_{i}}^{T+t_{i}}\left(C_{1}\left(a ; x_{a v g}^{\prime}\right)_{t_{i}}^{T+t_{i}}-C_{1}\left(\tilde{a} ; x_{a v g}^{\prime}\right)_{t_{i}}^{T+t_{i}}\right)}{2\left(\bar{a}_{x}^{t_{i}, T+t_{i}}\right)^{5} T^{3}}\right] \\
& +\mathcal{O}\left(\mathcal{M}_{1}(a)\left[\mathcal{M}_{0}(a)\right]^{2} t_{i}\right) .
\end{aligned}
$$

We conclude with an application of Lemma 1.3.

$\triangleright$ Step 3: Final residuals. Last, we let the reader verify that:

$$
\begin{aligned}
& \operatorname{Vega}^{\mathrm{BS}}\left(0,\left(\pi^{k}\left(a ; x_{a v g}^{\prime}\right)_{t_{i}}^{T+t_{i}}\right)^{2} T, k\right) \\
& \times \mathbb{E}\left[\pi_{0}\left(a ; k^{\prime}+X_{t_{i}}\right)_{t_{i}}^{T+t_{i}}-\bar{a}_{k^{\prime}+X_{t_{i}}}^{t_{i}, T+t_{i}}-\pi_{0}\left(a ; x_{a v g}^{\prime}\right)_{t_{i}}^{T+t_{i}}+\bar{a}_{x_{a v g}^{\prime} t_{i}, T+t_{i}}^{\prime}+k^{2}\left(\pi_{2}\left(a ; k^{\prime}+X_{t_{i}}\right)_{t_{i}}^{T+t_{i}}-\pi_{2}\left(a ; x_{a v g}^{\prime}\right)_{t_{i}}^{T+t_{i}}\right)\right] \\
\leq & \mathcal{M}_{1}(a)\left[\mathcal{M}_{0}(a)\right]^{3} \sqrt{T}\left(\sqrt{t_{i}}+\sqrt{T}\right)^{3}, \\
& \operatorname{Vomma}^{\mathrm{BS}}\left(0,\left(\pi^{k}\left(a ; x_{a v g}^{\prime}\right)_{t_{i}}^{T+t_{i}}\right)^{2} T, k\right) \mathbb{E}\left[\left(\pi^{k}\left(a ; k^{\prime}+X_{t_{i}}\right)_{t_{i}}^{T+t_{i}}-\pi^{k}\left(a ; x_{a v g}^{\prime}\right)_{t_{i}}^{T+t_{i}}\right)^{2}-\left(\bar{a}_{k^{\prime}+X_{t_{i}}}^{t_{i}, T+t_{i}}-\bar{a}_{x_{a v g}^{\prime}}^{t_{i}, T+t_{i}}\right)^{2}\right] \\
\leq & \mathcal{M}_{1}(a)\left[\mathcal{M}_{0}(a)\right]^{3} \sqrt{T}\left(\sqrt{t_{i}}+\sqrt{T}\right)^{3} .
\end{aligned}
$$

Combining (9)-(10)-(11)-(12)-(13)-(18)-(23)-(24) and identity (3) yields that:

$$
\begin{aligned}
& \operatorname{Call}^{\mathrm{FS}}\left(e^{x_{0}}, t_{i}, T, e^{k}\right) \\
= & \operatorname{Call}^{\mathrm{BS}}\left(0,\left(\pi^{k}\left(a ; x_{a v g}^{\prime}\right)_{t_{i}}^{T+t_{i}}\right)^{2} T, k\right)+\operatorname{Vega}^{\mathrm{BS}}\left(0,\left(\pi^{k}\left(a ; x_{a v g}^{\prime}\right)_{t_{i}}^{T+t_{i}}\right)^{2} T, k\right) \pi_{\mathrm{F}}^{k}\left(a ; x_{a v g}^{\prime}\right)_{t_{i}}^{T+t_{i}} \\
& +\frac{1}{2} \operatorname{Vomma}^{\mathrm{BS}}\left(0,\left(\pi^{k}\left(a ; x_{a v g}^{\prime}\right)_{t_{i}}^{T+t_{i}}\right)^{2} T, k\right)\left(\frac{C_{7}\left(a ; x_{a v g}^{\prime}\right)_{t_{i}}^{T+t_{i}}}{2 \bar{a}_{x_{a v g}^{\prime}, T+t_{i}}^{\prime} T} \mathcal{V}\left(a ; x_{0}\right)_{0}^{t_{i}}\right)^{2}+\mathcal{O}\left(\mathcal{M}_{1}(a)\left[\mathcal{M}_{0}(a)\right]^{3} \sqrt{T}\left(\sqrt{t_{i}}+\sqrt{T}\right)^{3}\right),
\end{aligned}
$$


Besides a Taylor expansion of Call ${ }^{\mathrm{BS}}\left(0,\left(\tilde{\sigma}_{\mathrm{I}, \mathrm{F}}^{3, x^{\prime}}{ }^{\prime}\left(x_{0}, t_{i}, T, k\right)\right)^{2} T, k\right)$ around $\pi^{k}\left(a ; x_{\text {avg }}^{\prime}\right)_{t_{i}}^{T+t_{i}}$ and Lemma 1.3 gives:

$$
\begin{aligned}
& \operatorname{Call}^{\mathrm{BS}}\left(0,\left(\tilde{\sigma}_{\mathrm{I}, \mathrm{F}}^{3, x_{a v g}^{\prime}}\left(x_{0}, t_{i}, T, k\right)\right)^{2} T, k\right) \\
= & \operatorname{Call}^{\mathrm{BS}}\left(0,\left(\pi^{k}\left(a ; x_{a v g}^{\prime}\right)_{t_{i}}^{T+t_{i}}\right)^{2} T, k\right)+\operatorname{Vega}^{\mathrm{BS}}\left(0,\left(\pi^{k}\left(a ; x_{a v g}^{\prime}\right)_{t_{i}}^{T+t_{i}}\right)^{2} T, k\right) \pi_{\mathrm{F}}^{k}\left(a ; x_{\text {avg }}^{\prime}\right)_{t_{i}}^{T+t_{i}} \\
& +\frac{1}{2} \operatorname{Vomma}^{\mathrm{BS}}\left(0,\left(\pi^{k}\left(a ; x_{\text {avg }}^{\prime}\right)_{t_{i}}^{T+t_{i}}\right)^{2} T, k\right)\left(\frac{C_{7}\left(a ; x_{\text {avg }}^{\prime}\right)_{t_{i}}^{T+t_{i}}}{2 \bar{a}_{x_{\text {avg }}^{\prime}, T+t_{i}}^{\prime} T} \mathcal{V}\left(a ; x_{0}\right)_{0}^{t_{i}}\right)^{2}+\mathcal{O}\left(\mathcal{M}_{1}(a)\left[\mathcal{M}_{0}(a)\right]^{3} \sqrt{T}\left(\sqrt{t_{i}}+\sqrt{T}\right)^{3}\right),
\end{aligned}
$$

which terminates the proof.

\section{Numerical Experiments}

$\triangleright$ Model. We consider the time-homogeneous hyperbolic local volatility model which the dynamic is:

$$
\mathrm{d} S_{t}=\nu\left\{\frac{\left(1-\beta+\beta^{2}\right)}{\beta} S_{t}+\frac{(\beta-1)}{\beta}\left(\sqrt{S_{t}^{2}+\beta^{2}\left(1-S_{t}\right)^{2}}-\beta\right)\right\} \mathrm{d} W_{t}, S_{0}=1,
$$

with $\nu>0$ the level of volatility and $\beta \in[0,1]$ the skew parameter. It corresponds to the Black-Scholes model for $\beta=1$ and exhibits a skew for the implied volatility surface when $\beta \neq 1$. This model introduced in [11] behaves closely to the CEV model but presents the advantage to avoid zero to be an attainable boundary. Thus the payoff of forward start options is well defined. Although the hypotheses of boundedness and ellipticity are not fulfilled, we reasonably expect that our approximation formula remains valid for this model and we apply Theorem 2.1 considering the log-asset with local volatility given by $a(x)=\nu\left\{\frac{\left(1-\beta+\beta^{2}\right)}{\beta}+\frac{(\beta-1)}{\beta}\left(\sqrt{e^{2 x}+\beta^{2}\left(1-e^{x}\right)^{2}}-\beta\right) e^{-x}\right\}$. $\triangleright$ Set of parameters. For the numerical experiments, we choose the values $\nu=20 \%, \beta=0.5$ and we allow the maturities, the forward dates and the strikes to vary. We test the forward dates $1 \mathrm{M}, 3 \mathrm{M}$ and $1 \mathrm{Y}$ and the maturities $1 Y$ and $10 Y$. Then the strikes approximately behave as $e^{q \nu \sqrt{T}}$ where $q$ denotes the value of various quantiles of the standard Normal law (from $1 \%$ to $99 \%$ ) to cover around the money $(k \approx 0)$ as well far from the money $(|k|$ large) options. The set of strikes are given w.r.t. the set maturities in Table 1.

TABLE 1. Set of maturities and strikes for the numerical experiments

\begin{tabular}{|l|rrrrrrrrrrrrr|}
\hline $\mathrm{T} / \mathrm{K}$ & $1 \%$ & $5 \%$ & $10 \%$ & $20 \%$ & $30 \%$ & $40 \%$ & $50 \%$ & $60 \%$ & $70 \%$ & $80 \%$ & $90 \%$ & $95 \%$ & $99 \%$ \\
\hline $1 \mathrm{Y}$ & 0.55 & 0.65 & 0.75 & 0.80 & 0.90 & 0.95 & 1.00 & 1.05 & 1.15 & 1.25 & 1.40 & 1.50 & 1.80 \\
$10 \mathrm{Y}$ & 0.15 & 0.25 & 0.35 & 0.50 & 0.65 & 0.80 & 1.00 & 1.20 & 1.50 & 1.95 & 2.75 & 3.65 & 6.30 \\
\hline
\end{tabular}

$\triangleright$ benchmark. As a benchmark, we use Monte Carlo methods with a variance reduction technique (see chapter 4 in [8] for example). The simulated variable is $\left(e^{X_{t_{i}+T}-X_{t_{i}}}-K\right)_{+}$and we use the Black-Scholes control variate $\left(e^{X_{t_{i}+T}^{\mathrm{BS}}-X_{t_{i}}^{\mathrm{BS}}}-K\right)_{+}-\mathbb{E}\left[\left(e^{X_{t_{i}+T}^{\mathrm{BS}}-X_{t_{i}}^{\mathrm{BS}}}-K\right)_{+}\right]$where $X_{t}^{\mathrm{BS}}=x_{0}+\nu W_{t}-\frac{1}{2} \nu^{2} t$, the latter expectation being computed with the Black-Scholes formula. Then we translate the results in terms of implied volatilities. We execute the simulations with $10^{7}$ sample paths and a time discretization of 300 steps per year using $\mathrm{C}++$ on a Intel(R) Core(TM) i5 CPU@2.40GHz with 4 GB of ram. We report in Table 2 the estimations of the forward implied volatilities obtained with Monte Carlo (MC) indicating the half-width of the related 95\%-symmetric confidence interval and with the third order approximation formula $(\operatorname{AF}(3))$. Sometimes for extreme strikes, we report ND in the tabular because the corresponding price approximation does not belong to the non-arbitrage interval.

$\triangleright$ Accuracy results. Globally the results are very good with a maximum error of around $50 \mathrm{bps}^{1}$ for $T=10 \mathrm{Y}$ and extreme strikes. But for such strikes, the MC estimate is questionable because of the large confidence

\footnotetext{
$1_{1} \mathrm{bp}$ (basis point) is equal to $0.01 \%$.
} 
intervals. The errors become higher when $t_{i}+T$ or $|k|$ increases and for $k \approx 0$ the results turn to be excellent with errors around $1 \mathrm{bp}$ or less for $T=1 \mathrm{Y}$ and around 3 or $4 \mathrm{bps}$ for $T=10 \mathrm{Y}$. Excepting the two extreme strikes, errors are generally smaller than 15 bps what is very satisfying. The quite important errors in extreme strikes are probably due to the Vega close to zero in these areas. We should mention that in term of prices, the approximations are extremely accurate with errors close to $1 \mathrm{bp}$ for the whole set of parameters.

TABLE 2. Hyperbolic model $(\beta=0.5, \nu=0.2)$ : forward implied volatilities in $\%$ with MC and AF(3).

\begin{tabular}{|c|c|c|c|c|c|c|c|c|c|c|c|c|c|c|c|}
\hline$T$ & $t_{i}$ & method $\backslash K$ & $1 \%$ & $5 \%$ & $10 \%$ & $20 \%$ & $30 \%$ & $40 \%$ & $50 \%$ & $60 \%$ & $70 \%$ & $80 \%$ & $90 \%$ & $95 \%$ & $99 \%$ \\
\hline \multirow[t]{9}{*}{$1 \mathrm{Y}$} & \multirow[t]{3}{*}{$1 \mathrm{M}$} & \multirow[t]{3}{*}{ MC } & 23.13 & 22.25 & 21.50 & 21.17 & 20.56 & 20.29 & 20.03 & 19.79 & 19.35 & 18.96 & 18.46 & 18.16 & 17.45 \\
\hline & & & \pm 0.09 & \pm 0.02 & \pm 0.01 & \pm 0.00 & \pm 0.00 & \pm 0.00 & \pm 0.00 & \pm 0.00 & \pm 0.00 & \pm 0.00 & \pm 0.01 & \pm 0.01 & \pm 0.14 \\
\hline & & & 23.14 & 22.26 & 21.51 & 21.17 & 20.57 & 20.29 & 20.03 & 19.79 & 19.35 & 18.95 & 18.43 & 18.13 & 17.37 \\
\hline & \multirow[t]{3}{*}{$3 \mathrm{M}$} & \multirow[t]{3}{*}{ MC } & 23.23 & 22.34 & 21.58 & 21.23 & 20.62 & 20.34 & 20.08 & 19.84 & 19.41 & 19.03 & 18.55 & 18.29 & 17.66 \\
\hline & & & \pm 0.10 & \pm 0.02 & \pm 0.01 & \pm 0.00 & \pm 0.00 & \pm 0.00 & \pm 0.00 & \pm 0.00 & \pm 0.00 & \pm 0.00 & \pm 0.01 & \pm 0.01 & \pm 0.12 \\
\hline & & & 23.31 & 22.38 & 21.59 & 21.24 & 20.62 & 20.34 & 20.08 & 19.84 & 19.41 & 19.02 & 18.53 & 18.25 & 17.57 \\
\hline & \multirow[t]{3}{*}{$1 \mathrm{Y}$} & \multirow[b]{3}{*}{$\mathrm{AF}$} & 23.85 & 22.82 & 21.92 & 21.53 & 20.86 & 20.57 & 20.30 & 20.07 & 19.67 & 19.35 & 18.99 & 18.83 & 18.50 \\
\hline & & & \pm 0.12 & \pm 0.03 & \pm 0.01 & \pm 0.01 & \pm 0.00 & \pm 0.00 & \pm 0.00 & \pm 0.00 & \pm 0.00 & \pm 0.00 & \pm 0.01 & \pm 0.01 & \pm 0.07 \\
\hline & & & 24.06 & 22.90 & 21.96 & 21.55 & 20.87 & 20.57 & 20.31 & 20.07 & 19.67 & 19.35 & 18.98 & 18.80 & 18.50 \\
\hline \multirow[t]{9}{*}{$10 \mathrm{Y}$} & \multirow[t]{3}{*}{$1 \mathrm{M}$} & \multirow[t]{3}{*}{ MC } & 28.50 & 26.56 & 25.14 & 23.50 & 22.22 & 21.19 & 20.09 & 19.21 & 18.17 & 17.04 & 15.74 & 14.78 & ND \\
\hline & & & \pm 0.22 & \pm 0.07 & \pm 0.04 & \pm 0.02 & \pm 0.01 & \pm 0.01 & \pm 0.01 & \pm 0.01 & \pm 0.01 & \pm 0.01 & \pm 0.03 & \pm 0.15 & ND \\
\hline & & & 27.83 & 26.33 & 25.08 & 23.51 & 22.24 & 21.21 & 20.10 & 19.21 & 18.17 & 17.04 & 15.71 & 14.76 & 13.29 \\
\hline & \multirow[t]{3}{*}{$3 \mathrm{M}$} & \multirow[t]{3}{*}{ MC } & 28.56 & 26.63 & 25.20 & 23.56 & 22.27 & 21.24 & 20.13 & 19.25 & 18.22 & 17.11 & 15.83 & 15.10 & ND \\
\hline & & & \pm 0.22 & \pm 0.07 & \pm 0.04 & \pm 0.02 & \pm 0.01 & \pm 0.01 & \pm 0.01 & \pm 0.01 & \pm 0.01 & \pm 0.01 & \pm 0.03 & \pm 0.12 & ND \\
\hline & & & 27.89 & 26.40 & 25.15 & 23.57 & 22.30 & 21.26 & 20.15 & 19.26 & 18.23 & 17.10 & 15.80 & 14.87 & 13.45 \\
\hline & \multirow[t]{3}{*}{$1 \mathrm{Y}$} & \multirow[b]{3}{*}{$\mathrm{AF}(3)$} & 28.80 & 26.87 & 25.44 & 23.77 & 22.47 & 21.43 & 20.33 & 19.46 & 18.46 & 17.39 & 16.21 & 15.55 & ND \\
\hline & & & \pm 0.22 & \pm 0.07 & \pm 0.04 & \pm 0.02 & \pm 0.01 & \pm 0.01 & \pm 0.01 & \pm 0.01 & \pm 0.01 & \pm 0.01 & \pm 0.03 & \pm 0.10 & ND \\
\hline & & & 28.18 & 26.70 & 25.43 & 23.83 & 22.54 & 21.48 & 20.37 & 19.49 & 18.48 & 17.40 & 16.20 & 15.38 & 14.17 \\
\hline
\end{tabular}

\section{REFERENCES}

[1] E. Benhamou, E. Gobet and M. Miri. Smart expansion and fast calibration for jump diffusion. Finance and Stochastics, 13(4): 563-589, 2009.

[2] L. Bergomi. Smile dynamics II. Risk, 67-73, 2005.

[3] L. Bergomi. Smile dynamics III. Risk, 90-96, 2008.

[4] P. Beyer and J. Kienitz. Pricing Forward Start Options in Models Based on (Time-Changed) Levy Processes. Preprint available on SSRN, 2008.

[5] R. Bompis and E. Gobet, Asymptotic and non asymptotic approximations for option valuation, in Recent Developments in Computational Finance: Foundations, Algorithms and Applications, T. Gerstner and P. Kloeden, eds., World Scientific Publishing Company, 2012.

[6] D. Brigo and F. Mercurio. Interest Rate Models Theory and Practice. Springer Finance, 2nd edition, 2006.

[7] J. Gatheral. The Volatility Surface, a Practioner's Guide. Wiley Finance, 2006.

[8] P. Glasserman. Monte Carlo Methods in Financial Engineering. Springer, 2003.

[9] P. S. Hagan, D. Kumar, A. S. Lesniewski, and D. E. Woodward. Managing smile risk. Willmott Magazine, 84-108, 2002.

[10] G. Hong. Forward Smile and Derivative Pricing. Preprint 2004.

[11] P. Jackel. Hyperbolic local volatility. Preprint, 2006.

[12] A. Jacquier and P. Roome. Asymptotics of forward implied volatility. Preprint available on arxiv, 2012.

[13] S. Kruse and U. Nogel. On the pricing of forward starting options in Hestons model on stochastic volatility. Finance and Stochastics, 9(2): 233-250, 2005.

[14] V. Lucic. Forward-start options in stochastic volatility models. Wilmott Magazine, 2003.

[15] D. Revuz and M. Yor. Continuous Martingales and Brownian Motion. Springer-Verlag, Hardcover, 3rd edition, 1999. 